\section{Measurement of anti-natalizumab antibodies by homogeneous mobility shift assay}

Sir,

We present a case of a multiple sclerosis (MS) patient who experienced an adverse event on her third infusion with natalizumab (Tysabri). Using the homogeneous mobility shift assay (HMSA), anti-drug antibodies (ADAs) to natalizumab were detected following second infusion. The adverse event experienced during third infusion coincides with measurement in vitro of ADA-drug complexes. Further treatment induced both an increase in ADA titre and in size of ADA-drug complexes formed.

While natalizumab is an effective therapeutic targeting integrin $\alpha 4 \beta 1$ for patients with MS there is a risk of developing immunoreactivity to the drug. Observational studies estimate the incidence of systemic hypersensitivity reactions as $4 \% .^{1}$ Hypersensitivity reactions associate with ADA and generally occur within 6 months of drug treatment. From 2006, the ADA enzyme linked immunoassay (ELISA) developed by Biogen Idec (USA) was integrated as part of clinical care in many countries. Studies report $9-14 \%$ of those receiving natalizumab developed ADAs when measured by Biogen ELISA and that ADAs associated with higher incidence of infusion-related reactions and reduced effectiveness of treatment. Natalizumab predominantly induces neutralising/idiotypic ADAs which reduce treatment effectiveness. $^{2-4}$ Another highly sensitive method now used for ADA detection is the Meso Scale Discovery platform (MSD; Meso Scale Diagnostics, USA) which identified high titre detection within 6 months of drug initiation as a predictor of ADA persistency. ${ }^{4}$ The aforementioned assays use ADAs to bridge between drug on a solid phase and labelled drug; this format may be prone to detection of low-affinity ADAs that do not compete with integrin $\alpha 4 \beta 1$ binding by natalizumab. Bridging assays may also fail to detect IgG4 ADA. ${ }^{5}$ The HMSA uses size exclusion chromatography to monitor in fluid phase ADAs bound or unbound to fluorescent-labelled drug. The HMSA method allows visualisation of the complexes of ADA-drug formed and thus confirms the avidity of all ADA isotypes for natalizumab.

Based on our previously described HMSA method, natalizumab was fluorescently labelled. ${ }^{6}$ Complexes of Alexa fluor 594-labelled natalizumab and ADA were separated from free fluorophore-labelled drug by HPLC size exclusion column chromatography and fluorescence detected. An acid dissociation step separates drug-ADA complexes making fluorescently labelled drug available to all ADAs present within the serum sample. In order to quantify the amount of ADA present, the total area under the size shifted fluorescent peaks was used to represent complexed drug and the area under the drug peak used to represent free drug. Drug naïve serum samples were used as negative controls.

This method was used to assess ADA in a 28-year-old female who was well after receiving two infusions of $300 \mathrm{mg}$ natalizumab, 4 weeks apart. No new neurological symptoms occurred before her third infusion 4 weeks later. After $40 \mathrm{~mL}$ of the $100 \mathrm{~mL}$ infusion she had chest tightness, facial swelling, hypertension, tachycardia and fever. She was treated intravenously with chlorpheniramine and hydrocortisone. Thereafter, she had no further natalizumab infusions and was started on alternate therapy. MS presents as relapses that may be months or years apart, so only if there had been a relapse could you potentially infer loss of effect of natalizumab. There was no relapse over her relatively short time on natalizumab. See Fig. 1 for a timeline diagram of infusions and sampling. Tryptase levels were not raised following the infusion reaction. Using an integrin $\alpha 4 \beta 1$ ELISA, natalizumab levels were measured and found to be low in all samples available (Table 1); low drug levels in S2 and S3 samples are suggestive of drug clearance by ADAs. The HMSA method demonstrates that the subject developed ADAs following the first two infusions (S2) which predominantly formed dimeric complexes with natalizumab in vitro (Fig. $2 \mathrm{~A}$ ), and it is these drug-ADA dimers that link with the adverse event experienced. One month after the third incomplete infusion (S3) the ADA response had progressed, the chromatogram providing a visual report of the in vitro change to formation of multimers of ADA-natalizumab complexes. A titration experiment indicated the ADA titre had increased (Fig. 2B). The presence of ADA to natalizumab was verified by the Biogen bridging ELISA on this sample (S3). Furthermore, using a competitive ligand binding assay, the ADA response had increased in neutralising capacity over time (Table 1 ). The competitive ligand binding assay detects the relative difference in integrin $\alpha 4 \beta 1$ between natalizumab spiked and un-spiked samples; any ADAs present in the sample interfere with natalizumab binding to integrin $\alpha 4 \beta 1$ and reduce the signal measured. A sample taken 6 months prior to receiving treatment was used as baseline.

Electron micrographs of infliximab-ADA complexes provide evidence that idiotypic ADAs limit the number of ADA molecules binding each $\mathrm{Fab}$ arm to one, forming dimeric, tetrameric and hexameric immune complexes of drug and ADA in equal ratio. ${ }^{7}$ Assuming an equal ratio of natalizumab to ADA in immune complexes formed, it is possible to

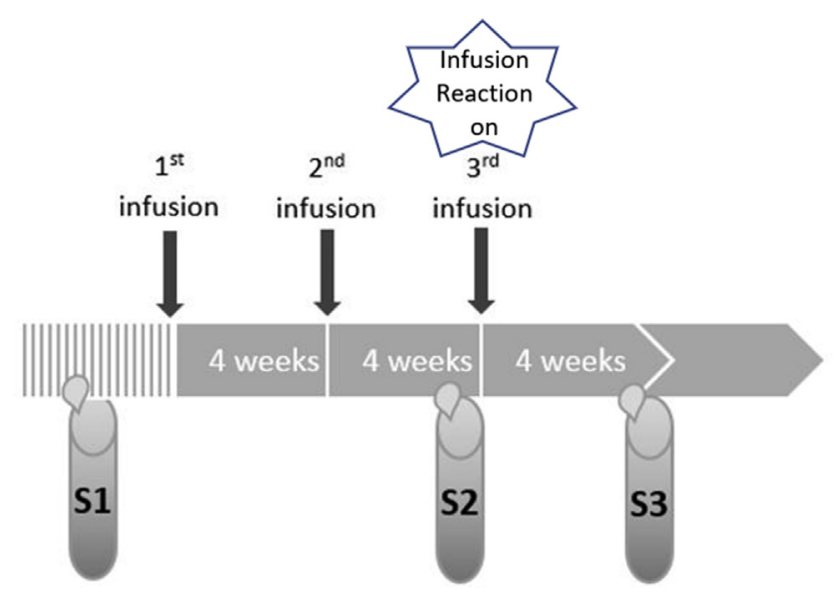

Fig. 1 Timeline of sampling and infusions. S1, 2 and 3 are serum samples collected, S1 being a baseline sample, S2 collected 1 hour before the third infusion and S3 taken 1 month after the third infusion. The patient had an adverse event on the third infusion. 
Table 1 Natalizumab serum concentration and ADA neutralising ability

\begin{tabular}{lllll}
\hline & $\begin{array}{c}\text { No. } \\
\text { natalizumab } \\
\text { infusions }\end{array}$ & $\begin{array}{c}\text { Biogen } \\
\text { ADA } \\
\text { bridging } \\
\text { ELISA }\end{array}$ & $\begin{array}{c}\text { Natalizumab } \\
\text { concentration } \\
(\mu \mathrm{g} / \mathrm{mL})^{\mathrm{a}}\end{array}$ & $\begin{array}{c}\text { ADA } \\
(\%)^{\mathrm{a}}\end{array}$ \\
\hline S1 (baseline) & 0 & Not tested & $<1.0$ & $6 \pm 10 \%$ \\
S2 & 3 & Not tested & $1.2 \pm 0.4$ & $71 \pm 12 \%$ \\
S3 & 3 & Positive & $<1.0$ & $96 \pm 5 \%$ \\
Positive & 9 & Positive & $<1.0$ & $97 \pm 4 \%$ \\
\multicolumn{1}{c}{ control $^{\mathrm{b}}$} & & & & \\
\hline
\end{tabular}

${ }^{\text {a }}$ Results expressed as $\bar{X} \pm S D(n=3)$.

$\mathrm{b}$ The positive control sample was also positive for ADA when measured on HMSA with multimeric drug-ADA complexes observed.
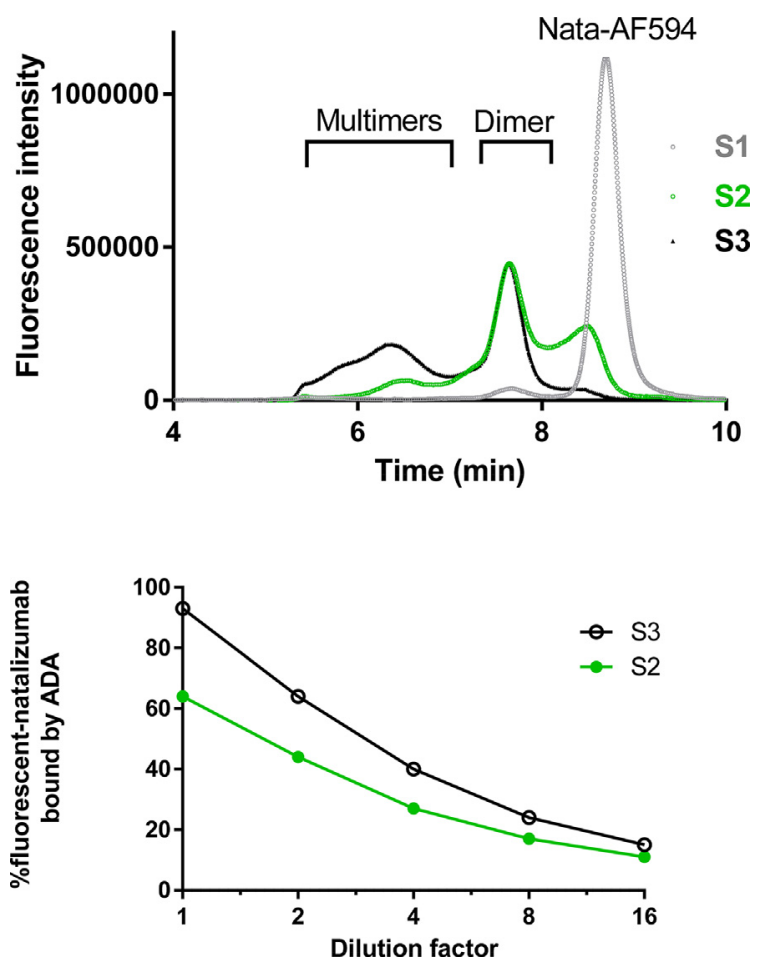

Fig. 2 Anti-natalizumab antibody measurement by HMSA. (A) Chromatograms obtained after size exclusion chromatography analysis of drug complex formation in patient sera from a baseline sample (S1), prior to third infusion (S2) and one month following third infusion (S3). Sera were acidified, then after neutralisation, incubated with Alexa Fluor594-natalizumab. Fluorescence units are expressed on the $\mathrm{y}$ axis and retention time in minutes on the $\mathrm{x}$ axis. Markers on the chromatogram indicate the retention time of fluorescent-labelled natalizumab monomer and ADA bound to fluorescent-labelled natalizumab as dimer or multimeric. The areas under the peaks in these regions were used to calculate the percentage of fluorescent-labelled natalizumab monomer bound in complex (complex/complex + free). (B) Titration of serum samples was used to calculate the concentration of ADA. A known amount of fluorescent-labelled natalizumab was added to the sample and ADA was calculated when $50 \%$ was bound by ADA. The concentration was $50 \%$ of $17 \mu \mathrm{g} / \mathrm{mL}$ multiplied by dilution factor, i.e., $15 \mu \mathrm{g} / \mathrm{mL}$ for $\mathrm{S} 2$ and $22 \mu \mathrm{g} / \mathrm{mL}$ for $\mathrm{S} 3$.

estimate the minimum ADA concentration. The samples were spiked with $17 \mu \mathrm{g} / \mathrm{mL}$ of labelled monomeric natalizumab and given the percentage shift of the monomeric drug into complexes, the ADA concentration was calculated at $15 \mu \mathrm{g} /$ $\mathrm{mL}$ prior to third infusion and $22 \mu \mathrm{g} / \mathrm{mL}$ one month afterwards (Fig. 2B). A recent study determined the mean trough concentration for those receiving natalizumab to be $26.1 \pm 14.1 \mu \mathrm{g} / \mathrm{mL}$ which would imply that the calculated
ADA concentration is within the expected range to neutralise drug. ${ }^{8}$ Thus, treatment boosted the ADA response in terms of increased ADA titre and size of immune complexes formed. Presence of multimeric complexes correlated with detection of neutralising ADA similar to our previous finding where the detection of multimeric infliximab-ADA complexes by HMSA selectively identified sera with neutralising capacity. The level of natalizumab in the sample series was low; again data from infliximab studies has shown that ADA detection by HMSA occurs at low drug levels. ${ }^{9}$

Completion of a clinical correlation or method comparison study to validate the method for use in clinical laboratories is our next step. This method can then be readily integrated into clinical laboratories routinely using HPLC and offers a quick turnaround time (20 minute runs) for results. In contrast to ELISA and MSD assays, the HMSA method provides information on concentration of ADA but also avidity in terms of immune complex sizes formed. The HMSA test may be useful in optimising the clinical benefit of natalizumab through identification of those at risk of ADAs and those with loss of response to drug as early as 2 months after initiating infusions.

Conflicts of interest and sources of funding: The authors state that there are no conflicts of interest to disclose.

\section{Paula E. Keating ${ }^{1}$, Roderick Duncan ${ }^{2}$, Myfanwy Spellerberg ${ }^{1}$, John O'Donnell ${ }^{1}$, Barry D. Hock}

${ }^{1}$ Immunology Section, Canterbury Health Laboratories, Christchurch, New Zealand; ${ }^{2}$ Department of Neurology, University of Otago, Christchurch, New Zealand; ${ }^{3}$ Haematology Research Group, Christchurch Hospital, New Zealand

Contact Paula Keating.

E-mail: paula.keating@cdhb.health.nz

1. Horga A, Castillo J, Rio J, et al. An observational study of the effec tiveness and safety of natalizumab in the treatment of multiple sclerosis Rev Neurol 2011; 52: 321-30.

2. Calabresi PA, Giovannoni G, Confavreux C, et al. The incidence and significance of anti-natalizumab antibodies: results from affirm and sentinel. Neurology 2007; 69: 1391-403.

3. Oliver B, Fernandez O, Orpez T, et al. Kinetics and incidence of antinatalizumab antibodies in multiple sclerosis patients on treatment for 18 months. Mult Scler 2011; 17: 368-71.

4. Deisenhammer F, Jank M, Lauren A, et al. Prediction of natalizumab antidrug antibodies persistency. Mult Scler 2019; 25: 392-8.

5. Bendtzen K. Anti-TNF-alpha biotherapies: perspectives for evidencebased personalized medicine. Immunotherapy 2012; 4: 1167-79.

6. Hock BD, McKenzie JL, Goddard L, et al. Discrimination of anti-drug antibodies with neutralizing capacity in infliximab- and adalimumabtreated patients: comparison of the homogeneous mobility shift assay and the affinity capture and elution assay. Therap Drug Monit 2018; 40: $705-15$.

7. van Schie KA, Kruithof S, Ooijevaar-de Heer P, et al. Restricted immune activation and internalisation of anti-idiotype complexes between drug and antidrug antibodies. Ann Rheum Dis 2018; 77: 1471-9.

8. Rispens T, Vennegoor A, Wolbink GJ, et al. Natalizumab remains detectable in patients with multiple sclerosis long after treatment is stopped. Mult Scler 2012; 18: 899-901.

9. Barclay ML, Karim S, Helms ETJ, et al. Infliximab and adalimumab concentrations and anti-drug antibodies in inflammatory bowel disease control using New Zealand assays. Intern Med J 2019; 49: 513-8.

DOI: https://doi.org/10.1016/j.pathol.2020.01.682 